\title{
Spectral condensation of turbulence in plasmas and fluids and its role in low-to-high phase transitions in toroidal plasma
}

\author{
M. G. Shats, H. Xia, and H. Punzmann \\ Research School of Physical Sciences and Engineering, Australian National University, Canberra, ACT 0200, Australia
}

(Received 18 August 2004; published 28 April 2005)

\begin{abstract}
Transitions from turbulence to order are studied experimentally in thin fluid layers and in magnetically confined toroidal plasma. It is shown that turbulence self-organizes through the mechanism of spectral condensation in both systems. The spectral redistribution of the turbulent energy leads to the reduction in the turbulence level, generation of coherent flow, reduction in the particle diffusion, and increase in the system's energy. The higher-order state in the plasma is sustained via the nonlocal spectral coupling of the linearly unstable spectral range to the large-scale mean flow. Spectral condensation of turbulence is discussed in terms of its role in the low-to-high confinement transitions in toroidal plasma which show similarity with phase transitions.
\end{abstract}

DOI: 10.1103/PhysRevE.71.046409

PACS number(s): 52.25.Gj, 05.65.+b, 03.75.Kk, 47.32.-y

\section{INTRODUCTION}

Turbulence determines properties of many natural and laboratory systems. Since its effects are often undesirable, the search for routes from turbulence to order is of great practical importance. Well-known examples of transitions from the turbulent to ordered state in plasma are low-to-high (L-H) confinement transitions in toroidal plasma devices. During these transitions the plasma evolves from the turbulent state, which is characterized by high fluctuation levels and anomalously high diffusion, to the state dominated by the increased plasma $(E \times B)$ flow, decreased turbulence level, and reduced diffusion. Though such transitions are often induced externally (for example, by increasing the heating power or by biasing the plasma using electrodes or limiters), spontaneous transitions are also commonly observed, suggesting that the external trigger is not generally necessary, as long as the plasma is close to the transition threshold. Extensive studies of this phenomenon for more than two decades, since the discovery of H mode in the ASDEX tokamak in 1982 [1], have revealed that the above features of $\mathrm{L}-\mathrm{H}$ transitions are universal in a variety of plasma devices, including tokamaks, stellarators, linear machines, and reversed-field pinches.

A number of theoretical models have been developed to explain L-H transitions [2], including those which consider the interaction between turbulence and plasma flows as the central point of this problem. Among them are theories in which plasma flows generated by turbulence are the essential component of the turbulence-flow interplay. In this contextnamely, in an attempt to find a self-consistent picture of the turbulence-flow interaction-the concept of zonal flows has received a renewed interest in plasma physics (for a review see, e.g., Ref. [3]). This has, in turn, attracted experimentalists trying to identify zonal flows in plasma experiments [4-8]. Zonal flows are poloidally and toroidally extended potential structures which are radially localized such that $k_{r}$ $\gg k_{\theta}=k_{\|}=0$ (where $k_{r}, k_{\theta}$, and $k_{\|}$are radial, poloidal, and parallel wave numbers correspondingly). The frequency of the zonal flow is somewhat less certain. Though zonal flows are generally thought to be the $f=0$ modes with a finite bandwidth, their other branch, the finite-frequency oscillating zonal flows, also termed geodesic acoustic modes, is also widely discussed. Regardless of the theoretical details, these anisotropic potential structures are believed to be generated by the small-scale unstable modes (e.g., drift waves) through wave-wave interactions and the spectral energy transfer from the higher- to the lower- $k$ spectral range (for example, through the inverse cascade). It is also expected that zonal flows inhibit radial particle transport and improve plasma confinement. Nevertheless, it is still not quite clear where the fundamental difference is between the zero-frequency zonal flow and the so-called mean shear flow, the difference sometimes stressed in theoretical works (e.g., Ref. [9]).

Despite large theoretical and experimental efforts to understand the mechanisms of the zonal flow generation and their effect on the plasma including their role in L-H transitions, progress is slowed down by limited diagnostic opportunities in plasmas and, first of all, in the visualization of turbulence and flows. As a result, measurements of the turbulence-flow interactions are often bound to be theory driven and, as such, are biased towards one model or another. Along with further diagnostic development and better visualization of turbulence, we propose a different approach in order to boost the construction of a consistent picture of the turbulence-flow interaction based on the inevitably fragmented experimental observations in plasmas. This approach is the comparative analysis of the hydrodynamic and plasma turbulence.

It should be noted that the generation of large-scale flows through their interaction with broadband turbulence is a subject which is not unique to plasma physics. The formation of large structures in two-dimensional (2D) turbulence has been extensively studied in neutral fluids since the 1960s, both theoretically and experimentally. We perform some of the fluid experiments with a particular emphasis on the generation of the large-scale flows and their interaction with smallscale turbulence. In this paper we compare some features of the evolution of the $2 \mathrm{D}$ fluid turbulence with results on the plasma turbulence modifications during L-H transitions in 
the $\mathrm{H}-1$ toroidal heliac and discuss new ideas on the generation of zonal flows and reduction of turbulence arising from such comparison. The comparison triggers useful analogies with regard to mechanisms of flow generation in plasmas, mostly thanks to the power of the 2D fluid flow visualization not available in plasma experiments. The comparison of the plasma turbulence with turbulence in thin layers of fluid is partially justified by extensive theoretical and modeling work done in the past on proving the analogy between the 2D hydrodynamic and toroidal plasma turbulence, in particular the similarity between the drift-wave turbulence in the Hasegawa-Mima model and the Rossby-wave turbulence in the Charney-Obukhov model (for a review see Ref. [10]). However, we stress that the fluid experiment presented here serves more as an inspiration for a first-principle interpretation of the plasma results rather than proof of our conclusions. On the other hand, similarities between the turbulence evolution in 2D fluid and in toroidal plasma, presented below, point to the universality of the self-organization in both systems.

The paper is organized as follows. In Sec. II we review some of the studies related to large-structure generation in 2D fluid turbulence and present experimental results on the spectral condensation of turbulence in thin layers of electrolyte in a cell. Section III describes the evolution of turbulent spectra measured in the low-temperature plasma of the H-1 heliac during spontaneous L-H transitions. The spectral energy transfer is analyzed which, together with the spectral energy balance estimates, leads to a conclusion about the spectral condensation of the turbulent energy. Finally, in Sec. IV we discus the results and summarize the conclusions.

\section{CONDENSATION OF SPECTRAL ENERGY IN 2D HYDRODYNAMIC TURBULENCE}

Studies of 2D turbulence were initially considered as a reduced problem which helped to better understand the practically more important 3D turbulent flows. It was realized, however, that 2D turbulence not only differs substantially from its 3D counterpart, but also may have a practical importance of its own. Though 2D flows do not, strictly speaking, exist, there are a number of examples of the flows in geophysics and astrophysics where quasi-2D turbulence serves as a good model. Here we summarize basic properties of the 2D turbulent flows before discussing experiments on condensation of the spectral energy.

It is well known that the evolution of spectra in 3D flows leads to the transfer of the spectral energy $E(k)$, towards smaller scales (larger $k$ ), a forward energy cascade, until it reaches the dissipation scale determined by the viscosity. Spectral regions of the energy injection $\left(k_{i}\right)$ and of the dissipation range $\left(k_{d}\right)$ do not generally coincide. The spectral range between them is called the inertial range. Viscous processes determine the energy dissipation rate $\varepsilon$ in the system. Kolmogorov [11] assumed that (a) the statistical properties of turbulence in the inertial range $\left(k \ll k_{d}\right)$ are determined only by $k$ and $\varepsilon$ and (b) $\varepsilon$ is the universal constant of a given flow in time and in space. Dimensional considerations have led to the famous Kolmogorov law for the spectral energy:

$$
E(k)=C \varepsilon^{2 / 3} k^{-5 / 3} .
$$

In 2D flows, in addition to the energy conservation, enstrophy, or the volume integral of the squared vorticity, $\Omega$ $=1 / 2 \int_{v} \omega^{2} d v$ (where $\omega=\boldsymbol{\nabla} \times V$ is the vorticity and $V$ is velocity) is also conserved [12]. The existence of this second invariant of the flow modifies the spectral transfer, which is now determined by both the energy $\varepsilon$ and the enstrophy $\varepsilon_{\omega}$, dissipation rates, and leads to the onset of two inertial ranges [13]. If energy and enstrophy are injected into the system at $k_{i}$, then energy in 2D flow cascades towards larger scales, or lower $k<k_{i}$ (inverse energy cascade range), while the enstrophy is transferred towards higher $k>k_{i}$ (forward enstrophy cascade). The former is described by the Kolmogorov law, Eq. (1) (though the energy is transferred in the opposite direction to that in the $3 \mathrm{D}$ turbulence), while the latter is described by

$$
E(k)=C_{\omega} \varepsilon_{\omega}{ }^{2 / 3} k^{-3} .
$$

The maximum of the energy spectrum thus lies in the low- $k$ range at $k_{E}$ and in the absence of the energy dissipation at large scales $k_{E}$ cannot be constant in time, $k_{E}=f(\varepsilon, t)$. If we introduce damping for large scales-for example, via linear damping $\mu$, the scale corresponding to the maximum of the spectrum-stabilizes at

$$
k_{E} \approx\left(\mu^{3} / \varepsilon\right)^{1 / 2} .
$$

If the system size is larger than this dissipation scale, $\lambda_{E}$ $=2 \pi / k_{E}$, one should observe the stationary spectrum showing two inertial ranges corresponding to the inverse energy cascade [Eq. (1)] and the direct enstrophy cascade [Eq. (2)]. For a detailed review on progress in the $2 \mathrm{D}$ turbulence studies see Ref. [14].

In this paper we will be mostly interested in conditions when the dissipative scale $\lambda_{E}$ exceeds the size of the experimental system, $\lambda_{c}$. In this case the spectral energy is accumulated at the largest scale allowed by the system size. Such a process, in which energy piles up in the largest scale $k_{c}$, was predicted by Kraichnan in 1967 who also noted the similarity between the condensation of the turbulent energy and the Bose-Einstein condensation of the 2D quantum gas [13]. The condensate formation in 2D fluids has been confirmed in experiments $[15,16]$ and in numerical simulations $[17,18]$.

The first convincing experimental evidence of the inverse energy cascade in 2D turbulence was presented by Sommeria in 1986 [15]. In this experiment, turbulence was generated in a thin layer of mercury in a cell. The fluid was placed in the vertical magnetic field. Thirty-six biased electrodes of varying polarity generated electric currents in a layer which, by interacting with the vertical magnetic field, generated 36 planar vortices in a cell. By varying the electric current and the depth of mercury layer, the forcing and linear damping could be finely controlled. Sommeria observed a $k^{-5 / 3}$ scaling due to the inverse cascade in the energy inertial range (though in a rather narrow $k$ range) and also reported the observation of the largest vortex limited by the cell size at low linear dissipation due to the process of spectral condensation. Another interesting observation which we will address in Sec. IV is 




FIG. 1. (Color online) Experimental setup for studying evolution of the $2 \mathrm{D}$ turbulence. A matrix of $10 \times 10$ magnetic dipoles (permanent magnets $4 \times 5 \times 10 \mathrm{~mm}^{3}, B=0.33 \mathrm{~T}$ on the surface of the magnet) is placed under the cell. An electric current flowing between the electrodes $(0.1-4 \mathrm{~A})$ interacts with the spatially varying magnetic field to generate $(J \times B)$-driven vortices (diameter of $\sim 12 \mathrm{~mm}$ ) above each of the magnetic dipoles.

that when the damping was close to some critical value, this largest vortex was observed to irregularly reverse its direction.

Detailed measurements of the spectral energy scaling were presented by Paret and Tabeling [16,19] in 1997-1998 in experiments in the stratified layers of electrolyte. In these experiments 2D turbulent flows were studied by generating $(J \times B)$-driven vortices in thin layers of fluid. The electric current of a random-in-time polarity flowing from one wall of the cell to another interacts with the spatially varying magnetic field to generate a matrix of vortices of varying signs. These experiments have reliably confirmed the existence of the inverse energy cascade in the quasi-2D turbulence, the $k^{-5 / 3}$ scaling, as predicted by Kraichnan [13], and also have confirmed the generation of the spectral condensate at low damping. In this paper we repeat with minor variations this experiment in order to obtain a more detailed description of the spectral condensation of turbulence than was previously published.

To generate the spectral condensate in a fluid we used experimental procedure reported by Paret and Tabeling [16], but with a constant instead of a random-in-time forcing. A turbulent flow is generated in a thin layer of electrolyte by driving $100(J \times B)$-forced vortices $(10 \times 10)$ in a cell. The schematic of the experimental setup is shown in Fig. 1. To study spectral condensation we are interested in the regime when the dissipation scale $\lambda_{E}$ [Eq. (3)] exceeds the size of the cell, $\lambda_{c}$. Since the dissipation scale is determined by the
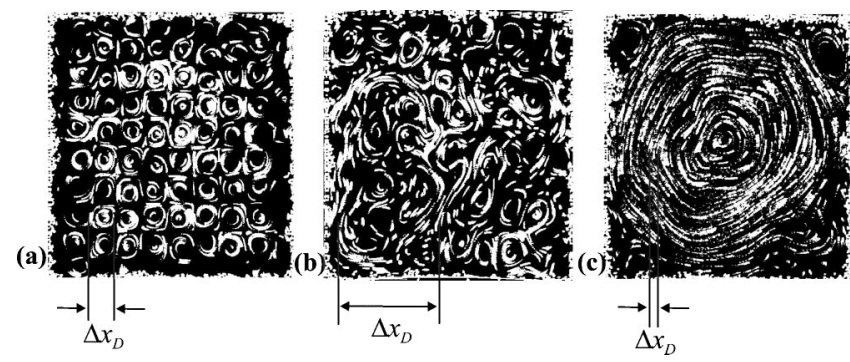

FIG. 2. Evolution of turbulence in a thin layer of electrolyte in a cell during spectral condensation. Trajectories of the tracer particles averaged over 12 frames of recorded video are shown. (a) The initial (linear) stage, $t=3 \mathrm{~s}$. (b) The inverse cascade stage, $t=25 \mathrm{~s}$. (c) The condensate stage, $t=60 \mathrm{~s} . \Delta x_{D}$ represents the spatial scale of the trace particle transport during three stages of the flow evolution.

frictional damping of the flow at the bottom wall $\mu$, two layers of electrolyte ( $\mathrm{NaCl}$ water solutions) of different concentrations (heavier solution at the bottom, total thickness of $6 \mathrm{~mm}$ ) are used to reduce $\mu$, as proposed in Ref [16]. The observation time is limited by the time it takes for the diffusion between the layers to destroy the stratification. Trajectories of the trace particles (latex particles, size $\sim 0.1 \mathrm{~mm}$ ) on the free surface of the fluid are recorded using a digital camera at 25 frames per second.

The evolution of the trace particle trajectories is shown in Fig. 2. After the force is applied (a dc current is turned on), three stages of the flow evolution are observed. At the early ("linear") stage, only vortices at the injection scale $k_{i}$, determined by the magnet sizes, are seen in Fig. 2(a). As the inverse energy cascade develops, the aggregation process drives larger structures [Fig. 2(b)]. After about $50 \mathrm{~s}$, global rotation dominates the flow [Fig. 2(c)] which then persists in a steady state. The total kinetic energy of the system increases from the linear stage to the inverse cascade stage until it reaches a maximum and stabilizes in the condensate regime as shown in Fig. 3(a). The kinetic energy is calculated from the velocity field, which is computed from the flow movie using a particle-image velocimetry technique.

The energy spectra of the velocity field are substantially modified during the system's evolution from the inverse cascade regime to the condensate stage [Fig. 3(b)]. As the system evolves, the turbulent energy is reduced over most of the $k$ range, except for the injection wave number $k_{i} \approx 630 \mathrm{~m}^{-1}$ and the smallest wave number $k_{c} \approx 80 \mathrm{~m}^{-1}$ determined by the size of the cell. It is this lowest mode at $k_{c}$ that is seen as the global rotation or condensate. A radial profile of the linear velocity of this largest vortex is shown in Fig. 3(c).

Scalings of the spectra in the inverse cascade and in the condensate regimes are shown in Fig. 4. In the inertial range corresponding to the enstrophy cascade $\left[k>k_{i}\right.$ in Fig. 4(a)], the spectrum scales as $k^{-3.5}$, which is slightly different than the scaling predicted by Kraichnan theory [13], probably for the reasons discussed in [20]. In the energy cascade inertial range $\left(k<k_{i}\right)$ the spectrum scales approximately as $k^{-5 / 3}$, though in our experiments this spectral range is not as smooth as in the experiments with the random-in-time forcing [19]. This shape of the spectrum changes dramatically after the spectral energy condenses into the largest vortex, as 


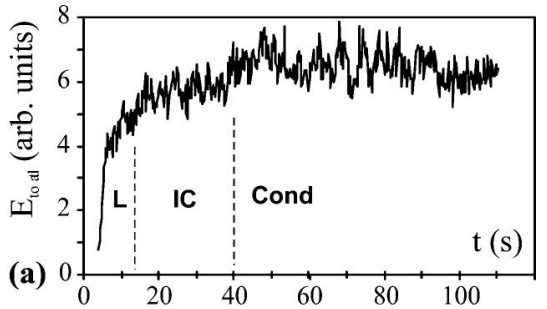

(b)

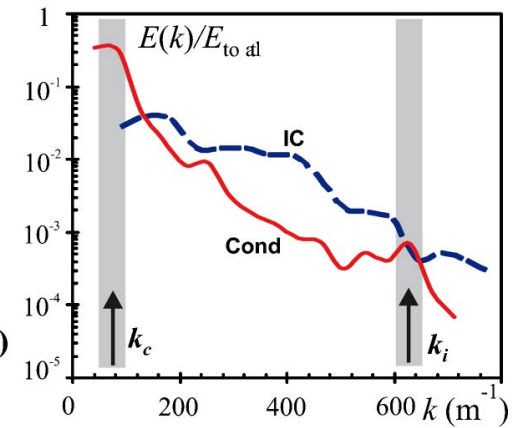

(c)

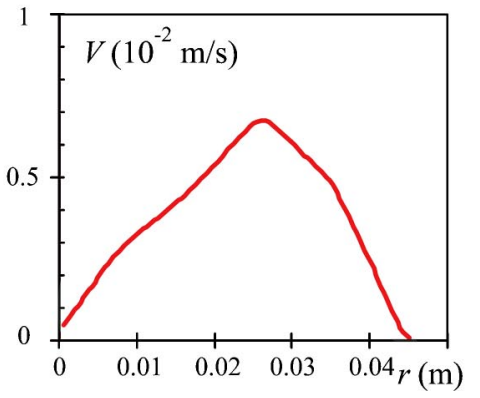

FIG. 3. (Color online) Analysis of the velocity field during spectral condensation in 2D fluid. (a) Temporal evolution of the total kinetic energy during the condensation. Three stages are marked: linear (L), inverse cascade (IC), and condensate (Cond). (b) Fluid turbulence spectra in the inverse cascade regime (dotted line) and in the condensate regime (solid line). Spectral energy is normalized by the total kinetic energy of the system. Shaded regions correspond to the injection scale $k_{i}$, and to the condensate scale $k_{c}$. (c) Linear velocity in the condensate vortex as a function of distance from the vortex center.

shown in Fig. 4(b). In this regime a $\sim k^{-3.3}$ scaling is observed both at $k<k_{i}$ and at $k>k_{i}$. A scaling close to $k^{-3}$ in the condensate has been noted by Tabeling [14]. Results presented here confirm the $k^{-3.3}$ scaling in the condensate which extends over two decades as seen in Fig. 4(b).

This strong deviation from the $k^{-5 / 3}$ law during the formation of the condensate is indicative of a change in the nature of the energy flow between the injection scale and the maximum of the spectrum. One may speculate that the spectral energy is transferred nonlocally from $k_{i}$ to $k_{c}$, skipping intermediate scales and thus leading to a reduction in the turbulence level in the inertial range seen in Fig. 3(b).

After the formation of the largest vortex, the condensate persists in a steady state. The energy necessary to overcome damping at this largest scale can only come from the energy source at $k_{i}$. This is possible if two spectral regions $k_{i}$ and $k_{c}$ are coupled such that the spectral energy is delivered from $k_{i}$ to $k_{c}$ directly, rather than through a multistep process of the energy cascade.

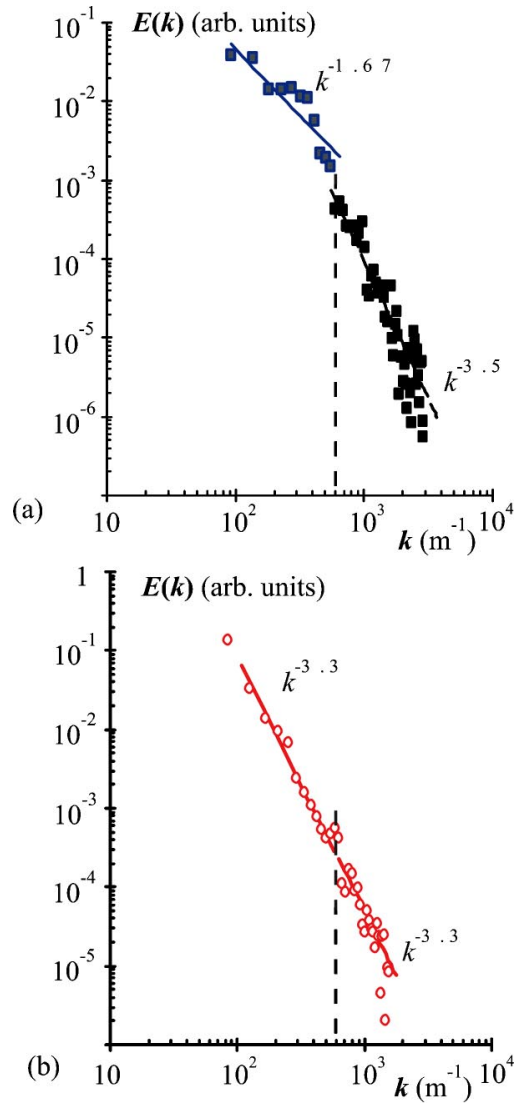

FIG. 4. (Color online) Energy spectra of the fluid velocities (a) during the inverse cascade stage of the flow development and (b) after the condensate has formed. The injection scale $k_{i}$ is shown by the vertical dashed line.

The establishment of the largest vortex greatly reduces the tracer particle diffusion (in fact, particle dispersion) in the system. As shown in Fig. 2, the "diffusion scale" $\Delta x_{D}$ increases from the linear stage to the inverse cascade regime. After the global flow is established, the diffusion scale drops, inhibiting the particle transport from the center of the largest vortex to its edge and thus reducing diffusion.

Summarizing the results of this section, under the conditions where damping due to the friction to the bottom is reduced and the dissipative scale exceeds the size of the cell, we observe spontaneous condensation of the spectral energy of turbulence into the large coherent vortex limited by the cell size (Fig. 2). During this process, at the constant energy input into the system, the total kinetic energy increases and reaches maximum in the condensate regime. The energy spectrum develops a strong peak at $k_{c}$ while the spectral energy at all intermediate scales between this maximum and the injection scale is reduced. This is seen as the disappearance of the Kolmogorov $k^{-5 / 3}$ inertial range and the onset of the $k^{-3.3}$ scaling. The trajectories of the trace particles change from chaotic in the inverse cascade phase of the flow evolution [Fig. 2(b)] to regular (deterministic) in the condensate regime [Fig. 2(c)]. This can also be interpreted as the reduction in trace particle dispersion in the condensate regime. 


\section{EVOLUTION OF THE PLASMA TURBULENCE SPECTRA DURING L-H TRANSITIONS}

Experimental studies of the density fluctuation spectra in magnetized plasma performed in 1970s and 1980s, mostly using microwave and laser scattering, revealed that these spectra are broad and have maxima at low frequencies and low wave numbers [21]. Since underlying linear instabilities suspected in driving the observed turbulent spectra, such as the drift-wave instabilities, are localized in a narrow higherfrequency spectral range, new theoretical models have been proposed to explain observations [10]. Hasegawa and Mima have shown that broadband spectra of the plasma drift-wave turbulence can be generated via three-wave interactions [22]. Then it was found that the structure of the Hasegawa-Mima equation, which describes the spectral evolution of the driftwave turbulence, is identical to the Charney equation describing the evolution of nonlinear Rossby waves in planetary atmospheres $[23,24]$. These models, similarly to the models of the 2D fluid turbulence described by the 2D Navier-Stokes equation, have two conserved quantities, energy and enstrophy, which lead to two inertial ranges and to the inverse cascade of energy in the turbulence spectra. Similarly to the fluid dynamics in 2D, condensation of the spectral energy in the largest scale has been predicted [23] and confirmed in numerical simulations [25]. In particular, such condensation has been shown to lead to the formation of zonal flows and other coherent structures which is a form of plasma self-organization.

Though many ingredients of the condensation process in plasma are known, there is no clear understanding how it is realized in magnetically confined plasma. As the terminology suggests, condensation is the process which leads to a phase transition. Here we attempt to put a more concrete meaning into this analogy based on an analysis of the turbulence spectra in plasmas close to and during transitions from low to high confinement mode. The term "phase transition" is more and more frequently used with regard to L-H transitions [26-30]. In this paper we propose a somewhat different interpretation of this analogy.

To analyze the turbulence condensation in plasma, spectral energy transfer during the turbulence evolution needs to be studied. Such an analysis is relatively easy to perform if a single-field description of plasma turbulence is valid. The mode-coupling mechanism in the turbulence may arise from the $E \times B$ convection of the density fluctuations [31] or from the polarization drift, which both appear in the equation of the ion dynamics [22]. The problem can be reduced to a singe-field model if the electron response to the potential fluctuations is adiabatic. In this case plasma turbulence can be modeled by the Charney-Hasegawa-Mima (CHM) model $[22,10]$, which also describes atmospheric turbulence. In the reported experiments in $\mathrm{H}-1$ at large ion gyroradii (due to high argon ion mass and low magnetic field), the polarization drift nonlinearity dominates so that the CHM model is applicable to our experiments [32,33]. This gives the opportunity to (a) study spectral transfer in a single-field model [34] and (b) partially justify the comparison of the spectra evolution in 2D fluid experiments with plasma results.

Plasma turbulence spectra discussed in this paper were obtained in the H-1 toroidal heliac [35], a three-field-period

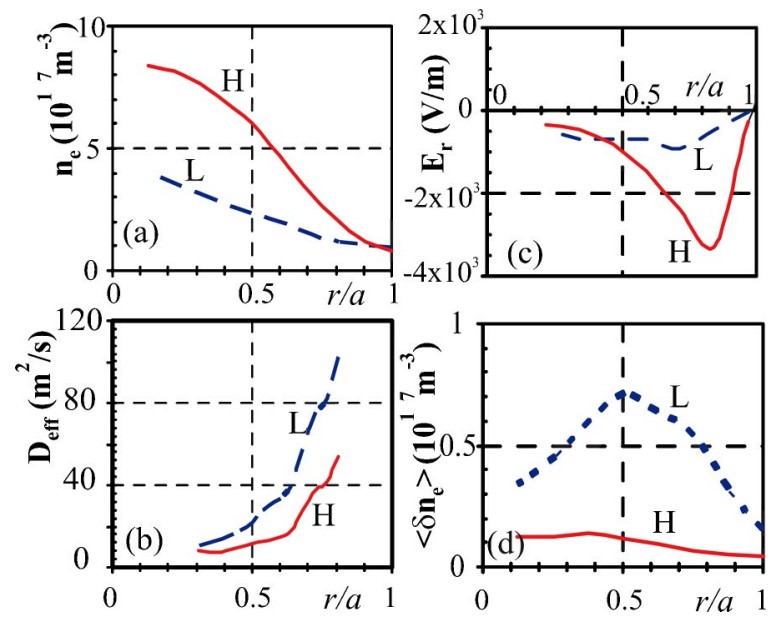

FIG. 5. (Color online) Radial profiles of the plasma parameters in $\mathrm{L}$ (dashed line) and $\mathrm{H}$ (solid line) modes: (a) electron density, (b) effective diffusion coefficient $D_{e f f}=\Gamma_{e} / \nabla n$, (c) radial electric field, and (d) root mean square of the density fluctuations.

helical axis stellarator, which has a major radius of $R=1 \mathrm{~m}$, and a mean minor radius of about $\langle a\rangle \approx 0.2 \mathrm{~m}$. Experiments are performed at low magnetic field in the range of $B$ $=[0.05-0.15] \mathrm{T}$ in the plasma produced by $\sim 60 \mathrm{~kW}$ of radio-frequency waves at $7 \mathrm{MHz}$. Plasma parameters are as follows: $n_{e} \sim 1 \times 10^{18} \mathrm{~m}^{-3}, T_{e} \sim 10 \mathrm{eV}$, and $T_{i} \sim 40 \mathrm{eV}$ in argon at filling pressures of $\sim 3 \times 10^{-5}$ Torr $[36,37]$. Such plasma parameters make the $\mathrm{H}-1$ plasma dimensionally similar to the edge plasma of bigger toroidal experiments, as discussed in Ref. [38].

We study the evolution of the turbulence spectra in $\mathrm{H}-1$ in the range of plasma parameters where transitions from low to high confinement modes are observed. Plasma conditions and thresholds at which L-H transitions are observed in H-1 are described in Ref. [39]. We compare the turbulence characteristics in plasma discharges sufficiently close to the threshold conditions, where spontaneous $\mathrm{L}-\mathrm{H}$ transitions are often observed with no external parameter "forcing" the transition. Radial profiles of the plasma parameters in $\mathrm{L}$ and $\mathrm{H}$ modes are shown in Fig. 5. The electron density doubles during the transition, while its fluctuations and the effective diffusion coefficient are substantially decreased. The radial electric field is greatly increased in the outer half of the plasma radius, as seen in Fig. 5(c). Fluctuations in the plasma electrostatic potential are also decreased by more than two orders of magnitude from the $\mathrm{L}$ to $\mathrm{H}$ mode.

The spectrum of the potential fluctuations in the L mode shows low-frequency coherent structures and a broadband turbulence in the higher- $k$ range shown in Fig. 6(a). Frequency spectra are computed from the time series of electrostatic potential, and then they are rescaled into the wave number domain as $k=2 \pi f / V_{E \times B}$, $(f$ is the frequency). Such rescaling is justified in view of the experimentally confirmed linear $f(k)$ dependence [33]. During the L-H transition, the spectral power of the fluctuations is reduced in a broad spectral range, except for a spectral region around $k \approx 200 \mathrm{~m}^{-1}$. We also compute the nonlinear spectral energy transfer due to three-wave interactions using a single-field model of 


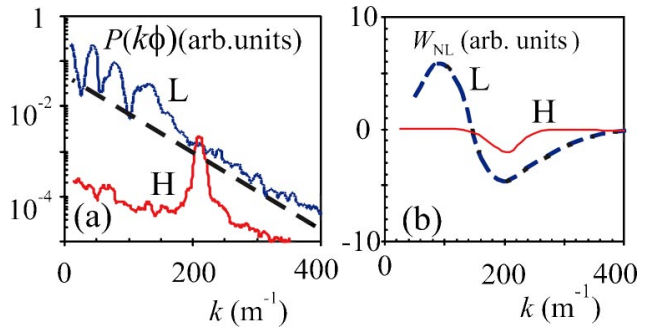

FIG. 6. (Color online) Modifications of the wave number spectra of the potential fluctuations in $\mathrm{L}$ and $\mathrm{H}$ modes: (a) power spectra of the floating potential fluctuations and (b) nonlinear energy transfer functions.

plasma turbulence as described in Ref. [33]. The nonlinear energy transfer function, which quantifies the energy exchanged between different waves due to the three-wave interactions, is computed as

$$
W_{N L}^{k} \approx\left(1+k_{\perp}^{2}\right) \sum_{k=k_{1}+k_{2}} T_{k}\left(k_{1}, k_{2}\right),
$$

where the spectral power transfer function $T_{k}$ is estimated from

$$
\frac{\partial P_{k}}{\partial t}=2 \gamma_{k} P_{k}+\sum_{k=k_{1}+k_{2}} T_{k}\left(k_{1}, k_{2}\right) .
$$

Equation (5) can be derived from the wave kinetic equation and solved for $T_{k}$ [33]. The nonlinear energy transfer function is shown in Fig. 6(b) for $\mathrm{L}$ and $\mathrm{H}$ modes.

In L mode, $W_{N L}^{k}$ is negative in the higher- $k$ range of $k$ $=(150-350) \mathrm{m}^{-1}$ and has a negative maximum at around $k_{i}$ $\approx 200 \mathrm{~m}^{-1}$. This spectral range loses on average spectral energy, which is gained by the lower- $k$ range characterized by positive $W_{N L}^{k}$. The spectral range around $k_{i} \approx 200 \mathrm{~m}^{-1}$ has been identified as that of the underlying linear instability [33]. The energy reservoir for this drift-wave-like instability is the plasma pressure gradient. Note that it is in this spectral range where fluctuations are not reduced during the confinement transition. This is in agreement with the fact that the energy reservoir for the instability does not decrease from the $\mathrm{L}$ to $\mathrm{H}$ mode, but rather increases due to the improved confinement and more peaked plasma pressure profiles. Thus, both the spectra evolution and the spectral transfer analysis identify the $k_{i} \approx 200 \mathrm{~m}^{-1}$ range as the linearly unstable spectral region. This range is equivalent to the injection scale in the hydrodynamic turbulence spectra discussed in Sec. II. Spectral energy is transferred from this scale towards lower wave numbers $k<k_{i}$ via the inverse energy cascade. The spectral range at higher wave numbers $k>k_{i}$ is the enstrophy-cascade range. In this range, spectra in L mode scale as $\sim k^{-3.9}$ as seen in Fig. 7. Such scaling is in good agreement with the shape of the spectra obtained in numerical simulations which show $\sim k^{-m}$, where $m \approx 4$ [10]. In $\mathrm{H}$ mode, the spectrum scales differently, showing $\sim k^{-3.0}$ at $k$ $>k_{i}$. Thus, the broadband spectrum is generated through a three-wave interaction process which leads to the inverse energy cascade and to the forward enstrophy cascade. The spectral transfer analysis results agree with theoretical pre-

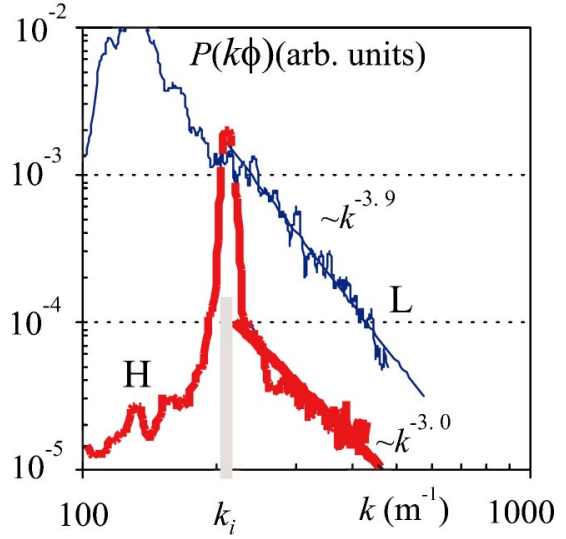

FIG. 7. (Color online) Scaling of spectra of the potential fluctuations in $\mathrm{L}$ and $\mathrm{H}$ modes in the enstrophy inertial range. $k_{i}$ denotes the spectral range of the underlying linear instability.

dictions with regard to the broadband part of the spectrum. Spectral energy transfer into the coherent spectral features seen in Fig. 6(a) will be discussed later.

Note that the spectral energy is reduced during L-H transitions both below and above $k_{i}$. This poses a question: where does the spectral energy of turbulence go during the transition? Our experimental data do not show any increase of the spectral energy at small scales. Following the fluid turbulence results of Sec. II, one may suggest that the spectral energy of the turbulence condenses into the lowest wave number, limited by the system size, and is observed as a large vortex, characterized by a stationary velocity profile, similar to that shown in Fig. 3(b). Such flow indeed develops during $\mathrm{L}-\mathrm{H}$ transitions and is observed as an increase in the mean $E \times B$ flow velocity [Fig. 8(b)], due to the increase in the radial electric field and coinciding with the reduction in turbulent fluctuations [Fig. 8(b)]. The electrostatic energy of the turbulent fluctuations $W_{T}=\int_{v} d \nu \Sigma_{k} m_{e} V_{k}^{2} / 2$ (where $\nu$ denotes volume and $V_{k}$ velocities associated with different turbulent scales $k$ ) should in this case transform into the kinetic energy of electrons (energy of the $E \times B$ flow) $W_{F}$ $=\int_{v} d \nu m_{e} V_{F}^{2} / 2$. The former can be estimated from experimental measurements of the potential fluctuation spectra by integrating over the plasma radius as

$$
W_{T} \sim \int_{0}^{a} r d r\left[\frac{n_{e L}}{B^{2}} \sum_{k=0}^{k_{i}} k^{2} \varphi_{k}^{2}\right],
$$

where $n_{e L}$ is the electron density in L mode, $V_{k}=k \phi_{k} / B$, and $B$ is the magnetic field. The increase in the energy of the flow during the $\mathrm{L}-\mathrm{H}$ transition is estimated as

$$
W_{F} \sim \int_{0}^{a} r d r\left[n_{e H} V_{H}^{2}-n_{e L} V_{L}^{2}\right]
$$

where $n_{e H}$ is the electron density in $\mathrm{H}$ mode and $V_{H}$ and $V_{L}$ are the $E \times B$ flow velocities in $\mathrm{L}$ and $\mathrm{H}$ modes correspondingly. These estimates of the turbulent and the flow energy agree in our experiment to within $20 \%$. This confirms that the observed increase in the $E \times B$ flow in $\mathrm{H}$ mode can be largely due to the redistribution of the spectral energy from 



FIG. 8. (Color online) (a) Spectra of the potential fluctuations and (b) radial profiles of the mean $E \times B$ flow velocity in $\mathrm{L}$ and $\mathrm{H}$ modes.

the intermediate scale into a mean zonal flow.

After the flow establishes in $\mathrm{H}$ mode, it should be sustained by the energy transfer from the unstable range at $k$ $\approx k_{i}$, similarly to the mechanism through which the mean flow of the condensate in 2D fluid is sustained by the force at the injection scale (see Sec. II). Below we present experimental evidence of such a spectral transfer in $\mathrm{H}$ mode.

The analysis of the spectral connection between the injection scale, $k \approx k_{i}$, and the condensate, $k \approx k_{c}$, denoted in Fig. 8 (a) as shaded areas, is performed in the time (frequency) domain using the amplitude correlation technique [40]. This technique has been successfully used to analyze the spectral transfer in the L-mode spectra in H-1 [33]. Two frequency bands of interest are selected from the fluctuation signal by bandpass-filtering it in order to obtain two time series. These two time series are then squared and passed through a lowpass filter to obtain only the slow varying amplitude information. Then the cross-correlation function between these signals is computed, to obtain the amplitude correlation function $(\mathrm{ACF})$

$$
F_{\mathrm{ACF}}(\tau)=\left\langle\left[x_{1}^{2}(t)\right]\left[x_{2}^{2}(t+\tau)\right]\right\rangle .
$$

The energy flow direction can then be determined from the sign of the time delay of the ACF. For example, if $x_{1}(t)$ and $x_{2}(t)$ correspond to the lower- and higher-frequency bands, correspondingly, and if the ACF has a maximum at the negative time lag, it means that the amplitude of the lowfrequency band lags with respect to the higher-frequency band. This is interpreted such that the low-frequency band receives its energy from the higher-frequency band.
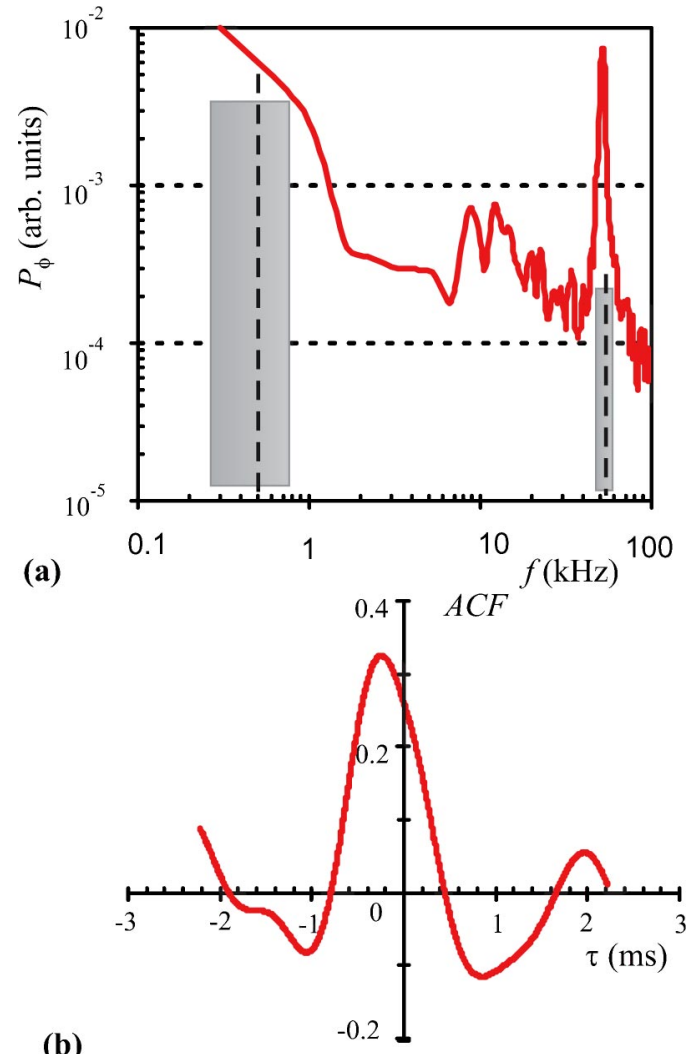

FIG. 9. (Color online) (a) Spectrum of the potential fluctuations in the $\mathrm{H}$ mode. Shaded areas show $\Delta f=0.5 \mathrm{kHz}$ wide frequency bands around the unstable range at $f \approx 50 \mathrm{kHz}$ and at $f \approx 0.5 \mathrm{kHz}$, within the spectral width of the mean flow. (b) Amplitude correlation function between the two $\Delta f=0.5 \mathrm{kHz}$ wide bands.

The frequency spectrum of the potential fluctuations in $\mathrm{H}$ mode is shown in Fig. 9(a). This spectrum corresponds to a "deep" $\mathrm{H}$ mode, obtained at higher magnetic field [36,39]. It is characterized by the broader (compared with $\mathrm{H}$ modes observed during spontaneous transitions) spectra both around the $f \approx 0$ and near the $f \approx 50 \mathrm{kHz}$ peak. The latter corresponds to the unstable (injection) range. The finite width of the two spectral ranges allows the amplitude correlation technique to be used as a diagnostic of the spectral coupling between these two regions. The amplitude correlation function $F_{\mathrm{ACF}}(\tau)$ between the $f \approx 0.5 \pm 0.25 \mathrm{kHz}$ and $f$ $\approx 50 \pm 0.25 \mathrm{kHz}$ bands is shown in Fig. 9(b). The ACF has a maximum of about 0.32 , suggesting substantial correlation between the two bands. A negative time lag (the ACF maximum corresponds to $\tau \approx-0.2 \mathrm{~ms}$ ) indicates that the spectral energy flows from the $f \approx 50 \mathrm{kHz}$ band into the $f \approx 0.5 \mathrm{kHz}$ band.

Summarizing results presented in this section, we have shown that the following.

(i) The broadband spectra in L mode are generated via three-wave interactions which lead to two spectral cascades: the inverse energy cascade and the forward enstrophy cascade.

(ii) Turbulence spectra evolve during L-H transitions such that the fluctuation level is reduced at both smaller and larger scales, while remaining approximately constant in the un- 
stable range (equivalent of the injection scale in 2D fluid experiment).

(iii) The reduction in the energy of the electrostatic turbulence during L-H transitions has the same order of magnitude as the increase in the energy of the mean $E \times B$ flow.

(iv) A finite-spectral-width, zero-frequency $E \times B$ flow (zonal flow) is spectrally coupled to the unstable range higher-frequency fluctuations in $\mathrm{H}$ mode such that the spectral energy flows from the higher-frequency range into the zonal flow.

Thus, we presented strong evidence that the mechanism of the reduction in turbulence coinciding with the increase in the $E \times B$ flow during L-H transitions is similar to the process of the spectral condensation of the turbulent energy in the 2D fluid described in Sec. II.

\section{DISCUSSION}

The turbulence evolution during the spectral condensation in 2D fluids presented in Sec. II is in many aspects similar to the modification of turbulence in toroidal plasma during spontaneous transitions from low- to high-confinement mode described in Sec. III. These similarities are summarized as follows.

(i) The inverse energy cascade leads to the generation of broad spectra and eventually to the condensation of spectral energy at the largest scale allowed by the system size.

(ii) The turbulence level is reduced at all scales between the injection scale and the condensate scale during spectral condensation. Two coupled but spectrally disconnected spectral regions are observed after the condensate formation: (a) coherent large-scale flow (zonal $E \times B$ flow in plasma) and (b) a smaller-scale spectral peak around the injection scale (linear instability range in plasma).

(iii) The nature of the spectral energy transfer from the injection scale towards large scales changes during the condensation process. This is seen as the disappearance of the $k^{-5 / 3}$ range in 2D fluids and as a strong reduction in the broadband turbulence in the plasma $\mathrm{H}$ mode.

(iv) The reduction in the particle diffusion is seen as the reduction in the effective diffusion coefficient in plasmas and as the modification of the tracer-particle orbits from chaotic to regular in $2 \mathrm{D}$ fluids.

Several of these observations are in agreement with theoretical models for the drift-wave turbulence by Balk et al. [41]. Using the hypothesis of the nonlocal inverse cascade, they predicted a reduction of the intermediate scales in the turbulent spectrum during the spectral condensation into the zonal flow. The splitting of the turbulent $k$ spectrum into two unconnected components-intensive zonal flow and a high- $k$ jet at the injection scale-was also shown. In this model a local (driven by comparable scales) inverse energy cascade leads to the generation of large structures. After reaching some threshold level, these structures force a change in the spectral transfer from the injection scale, which leads to a reduction in the intermediate scales $[42,43]$. After stabilization of the zonal flow, the energy transferred from the injection scale (high-frequency drift-wave jet) is much smaller than the energy of the zonal flow, but is sufficient to overcome dissipation at the condensate scale.
Theory of the nonlocal spectral transfer was first developed for the drift-wave turbulence using the CharneyHasegawa-Mima model [41-43]. Recently, a theory of the nonlocal turbulence described by the 2D Navier-Stokes equation has been proposed to describe the interaction of the coherent large-scale vortices with the broadband turbulence [44]. Theoretical predictions of the nonlocal turbulence theory (Fig. 7 of Ref. [44]) agree well with our result of Fig. 4(b) which shows a $k^{-3.3}$ scaling in the fluid condensate.

In our plasma experiments, the inverse energy cascade generates broadband turbulence in the L mode (Sec. III and $[32,33])$. After the transition to the $\mathrm{H}$ mode, the nonlocal spectral transfer from the unstable range is responsible for maintaining the zonal flow in the $\mathrm{H}$ mode, as follows from the result of Fig. 9. The nonlocal transfer means that the spectral energy is delivered to the condensate by the interacting wave triads such that $k_{c} \ll k_{1} \approx k_{2} \approx k_{i}$, where $k_{c}$ is the wave number of the condensate, $k_{c}=k_{1}-k_{2}$, and $k_{i}$ is the wave number of the injection scale.

In addition, it is appropriate to mention here that in lowconfinement mode, before L-H transitions, we observe both local and nonlocal inverse energy transfers in turbulent spectra, as described in Ref. [33]. The local energy cascade is responsible for the generation of the broadband spectra in $\mathrm{L}$ mode, while the nonlocal transfer is responsible for the coherent structure generation. In the plasma conditions close to the confinement transitions, large coherent structures having low poloidal mode numbers $m=0,1,2$, etc., are observed on top of the broadband spectra as seen in Fig. 6(a). Near the transition threshold, we observe the $m=0$ low-frequency (several kilohertz) mode identified as zonal flow [4], which has finite radial and nearly zero poloidal wave numbers, $k_{r}$ $\gg k_{\theta} \approx 0$. It is also driven from the unstable range via nonlocal coherent spectral transfer [33]. This regime, where the broadband turbulence coexists with coherent structures and oscillating zonal flows [33] has been identified as an intermediate mode, or I mode, and was likened to a metastable state near the phase transition threshold [38]. The term "I mode" is borrowed from Ref. [29] where the lowconfinement regime dominated by oscillating zonal flows is similarly described.

Further extending the phase transition analogy, we suggest that the plasma properties in the I mode may be considered as the incomplete condensation (metastable state). During the transition to the $\mathrm{H}$ mode, mean zonal flow (finitespectral-width $f=0$ mode) takes over the energy of the coherent structures and of the oscillating zonal flows. The latter thus act as nuclei in the phase transition. This is supported by our estimates of the turbulent energy and of the energy of the $E \times B$ flow in Sec. III. The role of zonal flows as of nuclei of the phase transitions has also been discussed from a different viewpoint in Ref. [38].

Oscillations of zonal flows near the threshold of spectral condensation are not unique for plasmas. It has already been mentioned in Sec. II that in 2D fluid irregular reversals of the global vortex were observed in experiments by Sommeria [15] when the linear damping was close to some critical value. Recently, a similar behavior of the $2 \mathrm{D}$ turbulence condensate has been found in a direct numerical simulation of the 2D turbulence by Molenaar et al. [45]. It is possible that 
such rotation reversal can be related to the critical behavior near the phase transition. Further experimental studies of the condensate formation in 2D fluids in the conditions where the dissipation scale [Eq. (3)] is close to the cell size will shed light on this problem.

Summarizing, the results discussed in this paper suggest that the low-to-high transitions in plasmas can be viewed as phase transitions in which the spectral condensation of turbulence plays a central role. In the frame of a qualitative model which can be built on the analogy with processes in the fluid turbulence, L-H transitions can be thought of as follows. The plasma pressure gradient is the energy reservoir for a drift-wave instability which develops in the plasma. The inverse energy and the forward enstrophy cascades lead to spectrum broadening such that its maximum shifts to larger and larger scales. At this stage large scales dominate the turbulence spectrum and the particle transport is hyperdiffusive. When the dissipative scale becomes comparable to the plasma minor radius, the spectral energy of turbulence is accumulated in the largest scale, leading to the formation of zonal flow. This coincides with the strong reduction in the fluctuation level everywhere in the spectrum, except for the linear-instability range and the condensate range. The turbulence energy transforms mostly into the energy of the mean $E \times B$ flow. As a result of the turbulence reduction, particle diffusion is also greatly reduced, leading, along with the increased $E_{r}$, to the confinement improvement in the $\mathrm{H}$ mode.
[1] F. Wagner et al., Phys. Rev. Lett. 49, 1408 (1982).

[2] J. W. Connor and H. R. Wilson, Plasma Phys. Controlled Fusion 42, R1 (2000).

[3] P. H. Diamond, K. Itoh, S.-I. Itoh, and T. S. Hahm, Plasma Phys. Controlled Fusion 47, R35 (2005).

[4] M. G. Shats and W. M. Solomon, Phys. Rev. Lett. 88, 045001 (2002).

[5] M. Jakubowski, R. J. Fonck, and G. R. McKee, Phys. Rev. Lett. 89, 265003 (2002).

[6] P. M. Schoch, K. A. Connor, D. R. Demers, and X. Zhang, Rev. Sci. Instrum. 74, 1846 (2003).

[7] A. Fujisawa et al., Phys. Rev. Lett. 93, 165002 (2004).

[8] G. R. Tynan et al., Phys. Plasmas 11, 5195 (2004).

[9] E. J. Kim and P. H. Diamond, Phys. Plasmas 11, L77 (2004).

[10] W. Horton and A. Hasegawa, Chaos 4, 227 (1994).

[11] A. N. Kolmogorov Dokl. Akad. Nauk SSSR 30, 301 (1941); 31, 538 (1941); 32, 16 (1941); in Turbulence, Classical Papers on Statistical Theory, edited by S. K. Friendlander and L. Topper (Interscience Publishers, London 1961).

[12] G. K. Batchelor, Phys. Fluids 12, 233 (1969).

[13] R. H. Kraichnan, Phys. Fluids 10, 1417 (1967).

[14] P. Tabeling, Phys. Rep. 362, 1 (2002).

[15] J. Sommeria, J. Fluid Mech. 170, 139 (1986).

[16] J. Paret and P. Tabeling, Phys. Fluids 10, 3126 (1998).

[17] M. Hossain, W. H. Matthaeu, and D. Montgomery, J. Plasma Phys. 30, 479 (1983).

[18] L. M. Smith and V. Yakhot, Phys. Rev. Lett. 71, 352 (1993).

[19] J. Paret and P. Tabeling, Phys. Rev. Lett. 79, 4162 (1997).

[20] V. Yakhot, Phys. Rev. Lett. 93, 014502 (2004).

[21] P. C. Liewer, Nucl. Fusion 25, 543 (1985).

[22] A. Hasegawa and K. Mima, Phys. Fluids 21, 87 (1978).

[23] A. Hasegawa, C. G. Maclennan, and Y. Kodama, Phys. Fluids 22, 2122 (1979).

[24] D. Fyfe and D. Montgomery, Phys. Fluids 22, 246 (1979).

[25] A. Hasegawa and M. Wakatani, Phys. Rev. Lett. 59, 1581
(1987).

[26] P. H. Diamond and Y. M. Liang, Phys. Rev. Lett. 72, 2565 (1994).

[27] B. A. Carreras, V. E. Lynch, L. Garcia, and P. H. Diamond, Phys. Plasmas 2, 2744 (1995).

[28] V. B. Lebedev and P. H. Diamond, Phys. Plasmas 4, 1087 (1997).

[29] R. J. Colchin et al., Phys. Rev. Lett. 88, 255002 (2002).

[30] D. del-Castillo-Negrete, B. A. Carreras, and V. E. Lynch, Plasma Phys. Controlled Fusion 46, A105 (2004).

[31] W. Horton, Phys. Rev. Lett. 37, 1269 (1976).

[32] H. Xia and M. G. Shats, Phys. Rev. Lett. 91, 155001 (2003).

[33] H. Xia and M. G. Shats, Phys. Plasmas 11, 561 (2004).

[34] Ch. P. Ritz and E. J. Powers, Physica D 20, 320 (1986).

[35] S. M. Hamberger, B. D. Blackwell, L. E. Sharp, and D. B. Shenton, Fusion Technol. 17, 123 (1990).

[36] M. G. Shats et al., Phys. Rev. Lett. 77, 4190 (1996).

[37] M. G. Shats, D. L. Rudakov, R. W. Boswell, and G. G. Borg, Phys. Plasmas 4, 3629 (1997).

[38] H. Punzmann and M. G. Shats, Phys. Rev. Lett. 93, 125003 (2004).

[39] M. G. Shats, C. A. Michael, D. L. Rudakov, and B. D. Blackwell, Phys. Plasmas 5, 2390 (1998).

[40] F. J. Crossley et al., Plasma Phys. Controlled Fusion 34, 235 (1992).

[41] A. M. Balk, V. E. Zakharov, and S. V. Nazarenko, Sov. Phys. JETP 71, 249 (1990).

[42] A. M. Balk, S. V. Nazarenko, and V. E. Zakharov, Phys. Lett. A 146, 217 (1990).

[43] A. I. Dyachenko, S. V. Nazarenko, and V. E. Zakharov, Phys. Lett. A 165, 330 (1992).

[44] S. Nazarenko and J.-P. Laval, J. Fluid Mech. 408, 301 (2000).

[45] D. Molenaar, H. J. H. Clercx, and G. J. F. van Heijst, Physica D 196, 329 (2004). 